\title{
Transfusion Management of a Patient with IgA Deficiency
}

\author{
Tejaswi Marri ${ }^{1}$, Allison K Buchanan ${ }^{2}$ and Ranu Jain ${ }^{2 *}$ \\ ${ }^{1}$ McGovern Medical School, Houston, USA \\ ${ }^{2}$ Department of Anesthesiology, The Fetal Center at Children's Memorial Hermann Hospital, McGovern Medical School, USA
}

*Corresponding author: Ranu R Jain, Associate Professor of Anesthesiology, The Fetal Center at Children's Memorial Hermann Hospital and Director of Pediatric Anesthesiology Fellowship, McGovern Medical School, Houston, TX, USA.

Received Date: December 08, 2019

Published Date: December 12, 2019

\section{Abstract}

Immunoglobulin (Ig) A deficiency is the most common primary immunodeficiency characterized by a decreased serum level of IgA in the presence of normal serum levels of other immunoglobulin isotypes. Patients with severe IgA deficiency may experience transfusion reactions when administered blood products that are not IgA deficient. There are complexities involved in organizing safe transfusion support, especially when the specific timing and transfusion needs of the procedure are unpredictable. While a history of IgA deficiency is certainly not a contraindication to surgical procedures requiring blood or blood product transfusions in the perioperative period, careful planning and a multidisciplinary approach are paramount in ensuring successful treatment as well as patient safety.

\section{Introduction}

Immunoglobulin (Ig) A deficiency is defined as the decreased serum level of IgA in the presence of normal serum levels of other immunoglobulin isotypes, IgG and IgM [1]. It is the most common primary immunodeficiency disease in patients greater than 4 years of age, with a reported frequency of 1/328 in the United States blood donors [2]. IgA is the most abundant antibody isotype produced in the body and it functions to protect against bacterial infections, especially in the pulmonary and digestive systems. While most patients who have IgA deficiency are asymptomatic, some patients develop allergies, recurrent sinopulmonary infections, chronic diarrhea, and lymphoid malignancies [1].

Patients with IgA deficiency, who have serum IgA levels less than $7 \mathrm{mg} / \mathrm{dL}$ may experience transfusion reactions to blood products which often contain small amounts of IgA [3]. Transfusion reactions may range in severity from minor reactions with signs and symptoms such as; fever, urticaria, chills, and pruritus to severe reactions presenting with more serious symptoms such as anaphylaxis, high fever, hypotension, respiratory distress, organ failure, and even death [3]. The cause of transfusion reactions in IgA deficient patients has not been conclusively demonstrated, however some studies have extrapolated that these reactions are due to antiIgA antibodies (IgG anti-IgA, IgE anti-IgA) [4]. IgA deficient patients requiring therapeutic transfusion or transfusion for major surgery must be screened carefully so that IgA reduced blood products are available [2].

\section{Discussion}

Preoperatively, patients with known IgA deficiency should be screened by thorough history and physical exam for symptomology of IgA deficiency including; recurrent respiratory tract infections, pneumonia, otitis, asthma, allergies, recurrent gastrointestinal infections and recurrent urinary tract infections [5]. Ideally, these conditions should be optimized prior to surgery.

Because of the association between anti-IgA antibodies and transfusion reactions in IgA deficient patients, current guidelines recommend screening for IgA antibodies in all patients with severe IgA deficiency or in patients who have experienced a transfusion reaction to a blood product in the past [6].

IgA deficient blood products are regarded as the "gold standard" in treatment/safety for these patients. In cases where blood and cellular products from IgA deficient donors is not readily available, washed blood cells and cellular products can be used [4]. Washing red blood cells (RBCs) with $0.9 \%$ saline and cycling three times removes $99 \%$ of IgA content from the product, effectively making 
the RBCs IgA deficient [7]. Autologous blood donation is also an option if cell salvage is available. Platelet products can be similarly washed and suspended in crystalloid solution $[4,8]$. Cryoprecipitate and fresh frozen plasma (FFP) obtained from IgA deficient donors can be stored frozen for 1 year and thawed 24 to 72 hours [9]. The goal is to avoid IgA containing products in patients with severe deficiency.

\section{Conclusion}

In conclusion, the providers should be cognizant of potential complications that can occur in patients with IgA deficiency when transfusing blood products. To safely manage these patients, a multidisciplinary approach is indispensable. Specific blood product availability should be discussed with the blood bank pathologists. The immunologists/ transfusion specialist should also be consulted [4]. In cases where emergent transfusion is needed, the patient may be pretreated with diphenhydramine and corticosteroids and administered standard blood products with very close/continuous monitoring $[4,10]$. An unknown risk of a transfusion reaction should never be a reason to withhold blood products in a life-threatening situation [10].

\section{Acknowledgement}

None.

\section{Conflict of Interest}

The authors report no conflict of interest.

\section{References}

1. Yel L (2016) Selective IgA deficiency. J Clin Immunol 30(1): 10-16.

2. Clark JA, Callicoat PA, Brenner NA, Bradley CA, Smith DM Jr (1983) Selective IgA deficiency in blood donors. Am J Clin Pathol 80(2): 210213.

3. Pineda AA, Taswell HF (1975) Transfusion reactions associated with anti-IgA antibodies: report of four cases and review of the literature. Transfusion 15(1): 10-15.

4. Davenport RD, Burnie KL, Barr RM (1992) Transfusion management of patients with IgA deficiency and anti-IgA during liver transplantation. Vox Sang 63(4): 247-250.

5. Latiff AHA, Kerr MA (2007) The clinical significance of immunoglobulin A deficiency. Ann Clin Biochem 44(Pt 2): 131-139.

6. Palmer DS, O’Toole J, Montreuil T, Scalia V, Yi QL, et al. (2010) Screening of Canadian Blood Services donors for severe immunoglobulin A deficiency. Transfusion 50(7): 1524-1531.

7. Tóth CB, Kramer J, Pinter J, Thek M, Szabó JE (1998) IgA Content of Washed Red Blood Cell Concentrates. Vox Sang 74(1): 13-14.

8. Sloand EM, Fox SM, Banks SM, Klein HG (1990) Preparation of IgAdeficient platelets. Transfusion 30(4): 322-326.

9. O’Shaughnessy DF, Atterbury C, Bolton Maggs P, Murphy M, Thomas D, et al. (2004) Guidelines for the use of fresh-frozen plasma, cryoprecipitate and cryosupernatant. Br J Haematol 126(1): 11-28.

10. Steel C, Vaida S, Mets B (2010) Case report: Massive blood transfusion in a patient with immunoglobulin a deficiency undergoing cesarean delivery. Anesth Analg 110(4): 1088-1090. 